Short Communication

\title{
How widespread is electronic cigarette use in outdoor settings? A field check from the TackSHS project in 11 European countries
}

\section{Beladenta Amalia ${ }^{1,2,3,4}$, Alejandro Rodríguez ${ }^{5}$, Elisabet Henderson ${ }^{5,6,7}$, Marcela Fu ${ }^{1,2,3,4}$, Xavier Continente ${ }^{5,7,8}$, Olena Tigova ${ }^{1,2,4}$, Sean Semple ${ }^{9}$, Luke Clancy ${ }^{10}$, Silvano Gallus ${ }^{11}$, Esteve Fernández ${ }^{1,2,3,4}$, Maria J. López ${ }^{5,6,7,8}$ and the TackSHS Project Investigators*}

${ }^{1}$ Tobacco Control Unit, Catalan Institute of Oncology (ICO), WHO Collaborating Centre for Tobacco Control, L'Hospitalet de Llobregat, Barcelona, Catalonia; ${ }^{2}$ Tobacco Control Research Group, Bellvitge Biomedical Research Institute (IDIBELL), L'Hospitalet de Llobregat, Barcelona, Catalonia;

${ }^{3}$ School of Medicine and Health Sciences, University of Barcelona, Barcelona, Spain; ${ }^{4}$ Consortium for Biomedical Research in Respiratory Diseases (CIBERES), Madrid, Spain; ${ }^{5}$ Agència de Salut Pública de Barcelona, Barcelona, Spain; ${ }^{6}$ Universitat Pompeu Fabra, Barcelona, Spain; ${ }^{7}$ Spanish Consortium for Research on Epidemiology and Public Health (CIBERESP), Madrid, Spain; ${ }^{8}$ Sant Pau Institute of Biomedical Research (IIB Sant Pau), Barcelona, Spain; ${ }^{9}$ Institute for Social Marketing, University of Stirling, Stirling, Scotland, United Kingdom; ${ }^{10}$ TobaccoFree Research Institute Ireland (TFRI), Dublin, Ireland; ${ }^{11}$ Istituto di Ricerche Farmacologiche Mario Negri IRCCS, Milan, Italy

*See full list of investigators at the end of manuscript

Corresponding author:

Dr. Esteve Fernández

Tobacco Control Unit, Catalan Institute of Oncology-ICO. Av. Granvia de l'Hospitalet, 199203, 08908. L'Hospitalet de Llobregat, Barcelona, Spain

E-mail : efernandez@iconcologia.net

Phone number : +34932607357 .

Word count $\quad: 1,731$

Abstract word count $\quad: 235$

References : 24

Numbers of figures and tables: 1 


\begin{abstract}
Exposure to secondhand aerosol from electronic cigarettes (e-cigarettes) may pose harms to bystanders, but they are used in many indoor settings. Less evidence exists on e-cigarette use in outdoor settings. This study aims to assess the use of e-cigarettes in outdoor settings in Europe. A cross-sectional study was conducted at the entrances of primary schools $(\mathrm{N}=200)$, children's playgrounds $(\mathrm{N}=200)$, and outdoor hospitality venues $(\mathrm{N}=220)$ during 2017-2018 in major cities of 11 European countries. We performed 30-minute observations and recorded e-cigarette use at three-time points: at 0 minutes, 15 minutes, and 30 minutes. We described the number and proportion of settings with e-cigarette use observed at any of the three-time points according to country and other contextual variables. Results showed that there were 22 (11.0\%) school entrances, eight (4.0\%) playgrounds, and 47 (21.3\%) outdoor hospitality venues where e-cigarette use was observed at any time point. School entrances and outdoor hospitality venues with observed e-cigarette use were more frequently found in countries with a higher prevalence $(\geq 1.4 \%)$ of e-cigarette use (school entrances: $18.0 \%$ vs. $4.0 \%$; $=0.002$, outdoor hospitality venues: $26.7 \%$ vs. $15.0 \%, \mathrm{p}=0.036$ ). In conclusion, the outdoor setting with the highest visibility of e-cigarette use was outdoor areas of hospitality venues. Although still limited, e-cigarettes were also used in outdoor settings frequented by children. Governments should consider measures to restrict e-cigarette use outdoors to protect the health of bystanders, particularly in areas where children may be present.
\end{abstract}

Keywords: e-cigarette, electronic nicotine delivery systems, passive exposure, outdoor settings 
Funding This project has received funding from the European Union's Horizon 2020 research and innovation programme under grant agreement No 681040. BA received the support of a fellowship from "La Caixa" Foundation (ID 100010434; Fellowship code: LCF/BQ/IN17/11620013). The Tobacco Control Research Group at ICO-IDIBELL (BA, EF, MF, OT) is partly supported by the Ministry of Universities and Research, Government of Catalonia (2017SGR319) and thanks CERCA Programme Generalitat de Catalunya for the institutional support to IDIBELL. EF is partly supported by the Instituto de Salud Carlos III, Government of Spain, co-funded by the European Regional Development Fund (FEDER; INT16/00211 and INT17/00103). This study is also supported by the Ministry of Universities and Research, Government of Catalonia under grant 2017SGR1526. The funders had no role in study design, data collection and analysis, decision to publish, or preparation of the manuscript. 


\section{INTRODUCTION}

Electronic cigarettes (e-cigarettes) have become popular in many parts of Europe; their use among adults increased from $12 \%$ in 2014 to $15 \%$ in $2017^{1}$. However, many toxic substances are present in e-cigarette aerosol ${ }^{2}$, posing potential risks to the health of users ${ }^{3}$ and non-users passively exposed to its secondhand aerosol (SHA $)^{4}$. Consequently, their use has been regulated in some indoor areas where the use of conventional tobacco products is already forbidden. Despite this, e-cigarette use has been observed in some smoke-free spaces in the United States (US) ${ }^{5}$, even where their use has been explicitly prohibited ${ }^{6}$. In outdoor spaces of dining areas and children's playgrounds in an Australian city, where tobacco smoking was forbidden, e-cigarette use was reported by $36.8 \%$ and $8.7 \%$ of e-cigarette users, respectively ${ }^{7}$.

As e-cigarette use has been widespread, exposure to SHA among bystanders merits some attention. About $16 \%$ of adults in the general population and $37 \%$ of adult smokers in Europe reported being exposed to SHA, mostly occurring in indoor areas of bars or restaurants and workplaces or educational venues ${ }^{8,9}$. In the US, SHA exposure in indoor and outdoor public places among middle and high school students surged, from $25.6 \%$ in 2017 to $33.2 \%$ in $2018^{10}$. In Europe, younger bystanders and those who live in countries with prevalent ecigarette use were more likely to be exposed to $\mathrm{SHA}^{8}$. Furthermore, seeing e-cigarette use in public places might renormalise smoking among youth ${ }^{11}$.

Given the increasing prohibitions of tobacco smoking outdoors and the bans on use of ecigarette indoors, e-cigarette use might be shifted to outdoor venues ${ }^{12}$. Furthermore, some of the health issues related to e-cigarette use indoors may also apply to outdoor areas, particularly where people are close together. Thus, an assessment of the extent of e-cigarette use outdoors is warranted; however, the evidence is absent in Europe.

This study aims to describe e-cigarette use in outdoor settings frequented by children or by large numbers of people, namely, school entrances, children's playgrounds, and outdoor hospitality venues in 11 European countries.

\section{MATERIALS AND METHODS}

This cross-sectional study, conducted within the framework of the TackSHS Project ${ }^{13}$, aimed to describe e-cigarette use outdoors in primary schools entrances, children's playgrounds, and outdoor hospitality venues, from March 2017 to October 2018, in major cities of 11 European countries: Bulgaria, France, Germany, Greece, Ireland, Italy, Poland, Portugal, Romania, 
Spain, and the United Kingdom (UK). The TackSHS project was approved by the Clinical Research Ethics Committee of the Bellvitge University Hospital (PR341/15), and the protocol of this study was approved by all the countries' local Research Ethics Committees.

Detailed methods have been reported elsewhere ${ }^{14}$. In brief, we selected densely populated urban areas in Bulgaria (Sofia), France (Paris), Germany (Mannheim, Heidelberg), Greece (Athens), Ireland (Dublin), Italy (Milano, Varese), Poland (Ciechanow, Warsaw), Portugal (Braga), Romania (Bucharest), Spain (Barcelona), and the United Kingdom (Edinburgh) based on opportunistic criteria, considering feasibility. From these cities, we conveniently selected 20 sites for each of the three types of outdoor settings in each country, except for France, where e-cigarette use data was only collected in outdoor hospitality venues. The selection of the sites considered neighbourhoods from different socioeconomic status (SES), which was assessed using local ecological synthetic indexes. When these synthetic indexes were not available, as was the case for Bulgaria, France, Greece, Poland, and Romania, other socioeconomic indicators were used, such as the cost of housing or the rate of poverty, among others. For each setting, half of the observations were made in the lowest SES neighbourhoods $\left(<20^{\text {th }}\right.$ percentile of the SES distribution) and the other half in the highest ( $>80^{\text {th }}$ percentile of the SES distribution). We visited a total of 200 school entrances, 200 children's playgrounds, and 220 outdoor hospitality venues across all countries.

Observations in school entrances and children's playgrounds were performed before the start or at the end of school hours. In outdoor hospitality venues (i.e., cafeterias, bars, and night pubs), half of the observations were made at any daytime and the other half after dinnertime during the weekdays and weekends. All observations started when at least five people, adults and/or children, were present in each setting.

The use of any type of electronic nicotine/non-nicotine delivery systems that vaporise liquid, hence, not heated tobacco products (e.g., iQOS), was recorded. A trained data collector recorded if e-cigarettes were used in each setting at the beginning ( 0 minutes), at 15 minutes, and at the end (30 minutes) of the observation period. We determined that an e-cigarette was used if data collectors visually noticed at least one person using e-cigarette at any of the three observation time points.

We reported the overall number and proportion (\%) of settings in which e-cigarettes were used, stratified by country, neighbourhood's SES, the country's tobacco control performance according to the Tobacco Control Scale score ${ }^{15}$, country's smoking prevalence ${ }^{16}$, country's e- 
cigarette use prevalence $^{13}$, and existence of national e-cigarette use regulation at the setting (obtained from different sources; see the footnote of Table 1). Chi-squared test was conducted to determine differences in proportions between subgroups at the 0.05 significance level. All analyses were performed with the statistical package Stata 15.

\section{RESULTS}

Overall, there were 22 out of $200(11.0 \%)$ school entrances in which e-cigarette use was observed (Table 1). While Greece had the highest proportion $(n=8 ; 40.0 \%)$, e-cigarette use was not observed at school entrances in Poland, Portugal, or Spain. School entrances with ecigarette use were observed four times $(18.0 \%$ vs. $4.0 \%$; $=0.002)$ more frequently in countries with higher $(\geq 1.4 \%)$ national prevalence of e-cigarette use than in countries with lower prevalence.

Table 1 also shows that e-cigarette use was observed in 8 out of 200 (4.0\%) children's playgrounds. Most of the venues were found in Ireland (3 playgrounds, 15.0\%), while ecigarette use was not observed in five countries (Bulgaria, Poland, Portugal, Spain, and the UK). No differences were found according to the studied contextual variables.

Finally, e-cigarette use was observed in 47 out of 220 (21.3\%) outdoor hospitality venues (Table 1), mainly in Greece and Portugal, in 9 (45.0\%) outdoor hospitality venues in each country. In contrast, e-cigarette use was not observed in any of the venues in Spain. Outdoor hospitality venues with e-cigarette use were more frequently observed in countries with a higher national prevalence of e-cigarette use $(26.7 \%$ vs. $15.0 \%, \mathrm{p}=0.036)$. 
Table 1. Number and proportion* of outdoor settings where electronic cigarette (e-cigarette) use was identified at any observation time ${ }^{1}$ according to contextual variables in 11 European countries. TackSHS project, 2017-2018.

\begin{tabular}{|c|c|c|c|c|c|c|}
\hline & \multicolumn{2}{|c|}{$\begin{array}{l}\text { SCHOOL } \\
\text { ENTRANCES } \\
(\mathbf{N}=\mathbf{2 0 0})\end{array}$} & \multicolumn{2}{|c|}{$\begin{array}{l}\text { CHILDREN'S } \\
\text { PLAYGROUNDS } \\
(\mathbf{N}=\mathbf{2 0 0 )}\end{array}$} & \multicolumn{2}{|c|}{$\begin{array}{c}\text { OUTDOOR HOSPITALITY } \\
\text { VENUES } \\
(\mathrm{N}=\mathbf{2 2 0})\end{array}$} \\
\hline & n (\%) & $p-$ value $^{2}$ & n (\%) & $p$-value ${ }^{2}$ & n (\%) & $p-$ value $^{2}$ \\
\hline All & $22(11.0)$ & & $8(4.0)$ & & $47(21.3)$ & \\
\hline Country & & - & & - & & - \\
\hline Bulgaria & $1(5.0)$ & & $0(0.0)$ & & $1(5.0)$ & \\
\hline France & - & & - & & $6(30.0)$ & \\
\hline Germany & $1(5.0)$ & & $1(5.0)$ & & $1(5.0)$ & \\
\hline Greece & $8(40.0)$ & & $1(5.0)$ & & $9(45.0)$ & \\
\hline Italy & $3(15.0)$ & & $1(5.0)$ & & $2(10.0)$ & \\
\hline Ireland & $4(20.0)$ & & $3(15.0)$ & & $6(30.0)$ & \\
\hline Poland & $0(0.0)$ & & $0(0.0)$ & & $3(15.0)$ & \\
\hline Portugal & $0(0.0)$ & & $0(0.0)$ & & $9(45.0)$ & \\
\hline Romania & $1(5.0)$ & & $2(10.0)$ & & $6(30.0)$ & \\
\hline Spain & $0(0.0)$ & & $0(0.0)$ & & $0(0.0)$ & \\
\hline United Kingdom & $4(20.0)$ & & $0(0.0)$ & & $4(20.0)$ & \\
\hline Neighbourhood socioeconomic status & & 0.175 & & 0.141 & & 0.173 \\
\hline High & $8(8.0)$ & & $2(2.0)$ & & $27(25.2)$ & \\
\hline Low & $14(14.0)$ & & $6(6.1)$ & & $20(17.7)$ & \\
\hline $\begin{array}{l}\text { Country's Tobacco Control Scale overall score } \\
(2016)^{3}\end{array}$ & & 0.094 & & 0.753 & & 0.502 \\
\hline$<50$ & $10(16.6)$ & & $2(3.3)$ & & $11(18.3)$ & \\
\hline$\geq 50$ & $12(8.5)$ & & $6(4.3)$ & & $36(22.5)$ & \\
\hline $\begin{array}{l}\text { Country's tobacco smoking prevalence } \\
(\%)^{4}\end{array}$ & & 0.651 & & 0.470 & & 0.076 \\
\hline$<31.0$ & $12(12.0)$ & & $5(5.0)$ & & $16(16.0)$ & \\
\hline$\geq 31.0$ & $10(10.0)$ & & $3(3.0)$ & & $31(25.8)$ & \\
\hline
\end{tabular}


$(\%)^{5}$

$$
<1.4
$$

$\geq 1.4$

$$
2(2.0)
$$

$215(15.0)$

$32(26.7)$

0.076

0.059 at the national-level ${ }^{6}$

Yes 3(5.0)

No
$0(0.0)$

$8(5.7)$

*Proportion was reported as a percentage among total observation in the corresponding contextual variable (by row).

${ }^{1} \mathrm{E}$-cigarette use data was collected over 30 minutes at three-time points: minute $0^{\prime}, 15^{\prime}$, and $30^{\prime}$.

${ }^{2}$ Chi-squared test.

${ }^{3}$ Tobacco Control Scale 2016 Ranking: <50 (Bulgaria, Germany, Greece) $\geq 50$ (France, Italy, Ireland, Poland, Portugal, Romania, Spain, UK).

${ }^{4}$ Current tobacco smoking prevalence (2017-2018, TackSHS survey data): $<31.0 \%$ (Germany, Ireland, Italy, Poland, and the United Kingdom) and $\geq 31.0 \%$ (Bulgaria, France, Greece, Portugal, Romania, and Spain). Cut-off was set at the median of total population current tobacco smoking prevalence across the listed countries (31.0\%).

${ }^{5}$ Current e-cigarette use prevalence (2017-2018, TackSHS survey data): $<1.4 \%$ (Germany, Italy, Poland, Portugal, and Spain) and $\geq 1.4 \%$ (Bulgaria, France, Greece, Ireland, Romania, and the United Kingdom). Cut-off was set at the median of total population e-cigarette use prevalence across the listed countries (1.4\%).

${ }^{6}$ Tobaccocontrollaws.org (accessed 20 May 2020), Globaltobaccocontrol.org (accessed 20 May 2020), and countries' national laws. Countries with e-cigarette use regulation in place for (a) School entrances: France, Italy, Poland, and Portugal; (b) Children's playgrounds: Poland, Portugal, and Spain; (c) Outdoor hospitality venues: none. 


\section{DISCUSSION}

Our findings show that e-cigarette use was observed in the three outdoor settings studied, even those frequented by children, with diversity across European countries. Some contextual factors at the national level can partly contribute to the intercountry differences observed. Country's e-cigarette prevalence, for example, was associated with observed e-cigarette use in school entrances and outdoor hospitality venues. Indeed, Greece, the UK, and Ireland, the countries with the highest proportion of school entrances with observed e-cigarette use, are countries with higher national e-cigarette use prevalence.

Our findings also indicate that e-cigarette use outdoors may happen regardless of the neighbourhood SES and the country's tobacco control performance. Previous studies suggested that e-cigarette use is not associated with individual's place of residence or $\mathrm{SES}^{17,18}$. Socioeconomic factors might play differently in e-cigarette use compared to tobacco smoking depending on attitudes and policies with regard to e-cigarette usage.

Since our study included areas frequented by adults and children, it suggests that the formulation of a comprehensive smoke-free policy should entail consideration of the impact of e-cigarette use on the perception of bystanders, in both population groups. There is evidence that SHA, including visibility of e-cigarette use, among adults may renormalise tobacco smoking, trigger relapse to smoking among quitters, and promote initiation of ecigarette use and, thus, put current smokers at risk of being dual users as they might start using e-cigarette ${ }^{19-21}$. A previous study has revealed that e-cigarette non-users who were current smokers or former e-cigarette users were more likely to be exposed by $\mathrm{SHA}^{8}$. Among youth, seeing e-cigarette use may normalise the use of nicotine-containing products, resulting in an increased risk of starting the use of e-cigarettes and tobacco products in the future ${ }^{10,11}$. Even among adolescents not susceptible to future cigarette smoking, exposure to the smell from e-cigarette use in indoor or outdoor public places was associated with susceptibility to using e-cigarettes ${ }^{22}$. As most of the current tobacco control policies tend not to restrict ecigarette use outdoors, it is advisable that their use is included in outdoor smoking restrictions, especially in areas where children may be present.

The scarcity of e-cigarette use observed in designated areas for children compared to outdoor hospitality venues, as also evident in the outdoor secondhand tobacco smoke exposure in 12 European countries, might be explained by the commonly perceived harms of SHA exposure 
for children ${ }^{23}$. However, the finding is likely to change over time with the increasing take-up of e-cigarettes, particularly to circumvent smoke-free regulations ${ }^{12}$. Having the same ecigarette use rules as for smoking in outdoor settings might provide simplicity in communication and implementation of the regulations.

The World Health Organization has advised countries to outlaw e-cigarette use in smoke-free places, including smoke-free areas outdoors, to protect non-users from SHA exposure ${ }^{24}$. Interestingly, we still observed some e-cigarette use activities in three school entrances where e-cigarette use was actually banned. The violation of the law highlights the importance of law enforcement.

Our study was limited by the convenience sampling of the settings selected and, thus, these results are not generalisable. Nevertheless, we monitored the main cities of 11 European countries, representative of different socio-cultural and socioeconomic characteristics. As the e-cigarette users in high SES areas can come from low SES areas, or vice versa, the stratification of settings by neighbourhood SES in this study should not be interpreted as the SES of e-cigarette users. Additionally, the difficult identification of e-cigarette use because of its similarity to other handheld items might influence our estimates. However, our data collectors were moving around the areas to maximise the observations. Given the limited duration of observation, our results might underestimate the real e-cigarette use in these settings; a longer observation period in future studies is warranted. Notwithstanding the limitations, this is the first multi-country study describing e-cigarette use in outdoor settings that considered different contextual determinants using a standardised protocol.

\section{CONCLUSIONS}

Our results show that e-cigarette use was observed in outdoor settings, including those frequented by children, across 11 European countries. Governments should consider strengthening their tobacco control policy by extending the smoke-free laws to cover ecigarette use in outdoor places, especially those where children are present and people are close together, as well as effectively enforcing the laws. 
Authors contribution Beladenta Amalia: Data curation, Writing-Original draft preparation. Alejandro Rodríguez: Investigation, Writing- Review \& Editing. Elisabet Henderson: Resources, Writing- Review \& Editing. Marcela Fu: Writing- Review \& Editing. Xavier Continente: Conceptualisation, Formal analysis, Writing- Review \& Editing. Olena Tigova: Writing- Review \& Editing. Sean Semple: Writing- Review \& Editing. Luke Clancy: Writing- Review \& Editing. Silvano Gallus: Writing- Review \& Editing. Esteve Fernández: Conceptualisation, Supervision, Writing- Review \& Editing. Maria J. López: Conceptualisation, Supervision, Writing- Review \& Editing

Acknowledgements We thank Ute Mons, Ramona Brad, Hristo Ivanov, Gergana Geshanova, Krzysztof Przewoźniak, José Precioso, and Joseph Osman for their contribution in coordinating data collection in the participating countries.

Competing Interests The authors declare no competing interests.

Disclaimer This paper reflects only the authors' views and the European Commission is not responsible for any use that may be made of the information it contains. 
*The TackSHS Project Investigators:

Catalan Institute of Oncology (ICO); Bellvitge Biomedical Research Institute (IDIBELL), Spain: Esteve Fernández, Yolanda Castellano, Marcela Fu, Montse Ballbè, Beladenta Amalia, Olena Tigova

Public Health Agency of Barcelona (ASPB), Spain: Maria José López, Xavier Continente, Teresa Arechavala, Elisabet Henderson

Istituto di Ricerche Farmacologiche Mario Negri IRCCS (IRFMN), Italy: Silvano Gallus, Alessandra Lugo, Xiaoqiu Liu, Elisa Borroni; Istituto DOXA, Worldwide Independent Network/Gallup International Association, Italy: Paolo Colombo

University of Stirling (UNISTIR), the UK: Sean Semple, Rachel O'Donnell, Ruaraidh Dobson

TobaccoFree Research Institute Ireland (TFRI), Ireland: Luke Clancy, Sheila Keogan, Hannah Byrne

Hellenic Cancer Society - George D. Behrakis Research Lab (HCS), Greece: Panagiotis Behrakis, Anna Tzortzi, Constantine Vardavas, Vergina Konstantina Vyzikidou, Gerasimos Bakelas, George Mattiampa

Fondazione IRCCS Istituto Nazionale dei Tumori (INT), Italy: Roberto Boffi, Ario Ruprecht, Cinzia De Marco, Alessandro Borgini, Chiara Veronese, Martina Bertoldi, Andrea Tittarelli

Istituto per lo Studio, la Prevenzione, e la Rete Oncologica (ISPRO), Italy: Giuseppe Gorini, Giulia Carreras, Barbara Cortini, Simona Verdi, Alessio Lachi, Elisabetta Chellini

Polytechnic University of Cartagena (UPCT), Spain: Ángel López Nicolás, Marta Trapero-Bertran, Daniel Celdrán Guerrero

European Network on Smoking and Tobacco Prevention (ENSP), Belgium: Cornel Radu-Loghin, Dominick Nguyen, Polina Starchenko

Fundación para la Investigación Biomédica del Hospital Universitario La Princesa (IISP), Spain: Joan B Soriano, Julio Ancochea, Tamara Alonso, María Teresa Pastor, Marta Erro, Ana Roca, Patricia Pérez, Elena García Castillo 


\section{REFERENCES}

1. European Comission. Special Eurobarometer 458 Report Attitudes of Europeans towards Tobacco and Electronic Cigarettes.; 2017. doi:10.2875/245123

2. National Academies of Sciences, Engineering and M. Public Health Consequences of E-Cigarettes. Vol 178. (Stratton K, Kwan LY, Eaton DL, eds.). Washington, D.C.: National Academies Press; 2018. doi:10.17226/24952

3. Bozier J, Chivers EK, Chapman DG, et al. The Evolving Landscape of e-Cigarettes. Chest. 2020;157(5):1362-1390. doi:10.1016/j.chest.2019.12.042

4. Bayly JE, Bernat D, Porter L, Choi K. Secondhand Exposure to Aerosols From Electronic Nicotine Delivery Systems and Asthma Exacerbations Among Youth With Asthma. Chest. 2019;155(1):88-93. doi:10.1016/j.chest.2018.10.005

5. Dunbar ZR, Giovino G, Wei B, O’Connor RJ, Goniewicz ML, Travers MJ. Use of Electronic Cigarettes in Smoke-Free Spaces by Smokers: Results from the 2014-2015 Population Assessment on Tobacco and Health Study. Int J Environ Res Public Health. 2020;17(3):978. doi:10.3390/ijerph17030978

6. Yingst JM, Lester C, Veldheer S, Allen SI, Du P, Foulds J. E-cigarette users commonly stealth vape in places where e-cigarette use is prohibited. Tob Control. 2019;28(5):493-497. doi:10.1136/tobaccocontrol-2018-054432

7. Twyman L, Watts C, Chapman K, Walsberger SC. Electronic cigarette use in New South Wales, Australia: reasons for use, place of purchase and use in enclosed and outdoor places. Aust N Z J Public Health. 2018;42(5):491-496. doi:10.1111/17536405.12822

8. Amalia B, Liu X, Lugo A, et al. Exposure to secondhand aerosol of electronic cigarettes in indoor settings in 12 European countries: data from the TackSHS survey. Tob Control. March 2020:tobaccocontrol-2019-055376. doi:10.1136/tobaccocontrol2019-055376

9. Tigova O, Beladenta A, Castellano Y, et al. Secondhand exposure to e-cigarette aerosols among smokers: A cross-sectional study in six European countries of the EUREST-PLUS ITC Europe Surveys. Tob Induc Dis. 2019;16(2).

doi:10.18332/tid/99117 
10. Dai H. Exposure to Secondhand Aerosol From Electronic Cigarettes Among US Youth From 2015 to 2018. JAMA Pediatr. 2020;174(3):298.

doi:10.1001/jamapediatrics.2019.5665

11. Agaku IT, Perks SN, Odani S, Glover-Kudon R. Associations between public ecigarette use and tobacco-related social norms among youth. Tob Control. 2020;29(3):332-340. doi:10.1136/tobaccocontrol-2018-054728

12. Patel D, Davis KC, Cox S, et al. Reasons for current E-cigarette use among U.S. adults. Prev Med (Baltim). 2016;93:14-20. doi:10.1016/j.ypmed.2016.09.011

13. Fernández E, López MJ, Gallus S, et al. Tackling second-hand exposure to tobacco smoke and aerosols of electronic cigarettes: the TackSHS project protocol. Gac Sanit. 2020;34(1):77-82. doi:10.1016/j.gaceta.2019.07.002

14. Henderson E, Continente X, Fernández E, et al. Secondhand smoke exposure and other signs of tobacco consumption at outdoor entrances of primary schools in 11 European countries. Sci Total Environ. 2020;743:140743. doi:10.1016/j.scitotenv.2020.140743

15. Joossens L, Raw M. The Tobacco Control Scale 2016 in Europe. Brussels; 2017. www.tobaccocontrolscale.com. Accessed July 1, 2019.

16. Gallus S, Lugo A, Liu X, et al. Who smokes in Europe? Data from 12 European countries in the TackSHS survey (2017-2018). J Epidemiol. 2020:JE20190344. doi:10.2188/jea.JE20190344

17. Vardavas CI, Filippidis FT, Agaku IT. Determinants and prevalence of e-cigarette use throughout the European Union: a secondary analysis of 26566 youth and adults from 27 Countries. Tob Control. 2015;24(5):442-448. doi:10.1136/tobaccocontrol-2013051394

18. Friedman AS, Horn SJL. Socioeconomic Disparities in Electronic Cigarette Use and Transitions from Smoking. Nicotine Tob Res. 2019;21(10):1363-1370. doi:10.1093/ntr/nty120

19. King AC, Smith LJ, Fridberg DJ, Matthews AK, McNamara PJ, Cao D. Exposure to electronic nicotine delivery systems (ENDS) visual imagery increases smoking urge and desire. Psychol Addict Behav. 2016;30(1):106-112. doi:10.1037/adb0000123

20. Mirbolouk M, Charkhchi P, Orimoloye OA, et al. E-Cigarette Use Without a History 
of Combustible Cigarette Smoking Among U.S. Adults: Behavioral Risk Factor Surveillance System, 2016. Ann Intern Med. 2019;170(1):76. doi:10.7326/M18-1826

21. King AC, Smith LJ, McNamara PJ, Matthews AK, Fridberg DJ. Passive exposure to electronic cigarette (e-cigarette) use increases desire for combustible and e-cigarettes in young adult smokers. Tob Control. 2015;24(5):501-504.

doi:10.1136/tobaccocontrol-2014-051563

22. Kowitt SD, Osman A, Ranney LM, Heck C, Goldstein AO. E-Cigarette Use Among Adolescents Not Susceptible to Using Cigarettes. Prev Chronic Dis.

2018;15(2):170368. doi:10.5888/pcd15.170368

23. Nguyen KH, Tong VT, Marynak K, King BA. Perceptions of Harm to Children Exposed to Secondhand Aerosol From Electronic Vapor Products, Styles Survey, 2015. Prev Chronic Dis. 2017;14:160567. doi:10.5888/pcd14.160567

24. The Regional Office for Europe of the World Health Organization. Electronic Nicotine and Non-Nicotine Delivery Systems a Brief.; 2020.

http://www.euro.who.int/pubrequest. Accessed May 26, 2020. 